\title{
Value of Bronchoscopy after EUS in the Preoperative Assessment of Patients with Esophageal Cancer at or Above the Carina
}

\author{
Jikke M. T. Omloo • Mark van Heijl • \\ Jacques J. G. H. M. Bergman • Mia G. J. Koolen • \\ Mark I. van Berge Henegouwen •
}

J. Jan B. van Lanschot

Received: 13 March 2008 / Accepted: 2 May 2008 /Published online: 5 June 2008

(C) 2008 The Author(s)

\begin{abstract}
Introduction Esophageal cancer is an aggressive disease with a strong tendency to infiltrate into surrounding structures. The aim of the present study is to determine the additional value of bronchoscopy for detecting invasion of the tracheobronchial tree after endoscopic ultrasonography (EUS) in the preoperative assessment of patients with esophageal cancer at or above the carina. Materials and Methods Between January 1997 and December 2006, 104 patients were analyzed for histologically proven esophageal cancer at or above the carina. All patients underwent both EUS and bronchoscopy (with biopsy on indication) in the preoperative assessment of local resectability.

Results and Discussion After extensive diagnostic workup, 58 of 104 patients (56\%) were eligible for potentially curative esophagectomy; nine of these 58 patients $(9 / 58,15 \%)$ appeared to be incurable peroperatively because of ingrowth in the tracheobronchial tree (five patients), ingrowth in other vital structures (two patients) or distant metastases (two patients). Of the 46 non-operable patients, local irresectability (T-stage 4 ) was identified in 26 patients $(26 / 46,57 \%$ ) due to invasion of vital structures on EUS: invasion of the aorta in six patients, invasion of the lung in 11 patients; in 12 patients invasion of the tracheobronchial tree was described, which was confirmed by bronchoscopy in only five patients. No patients with T4 were identified by bronchoscopy alone.

Conclusion For patients with esophageal tumors at or above the carina, no additional value of bronchoscopy (with biopsy on indication) to exclude invasion of the tracheobronchial tree was seen after EUS in a specialized centre. Although based on relatively small numbers, we conclude that bronchoscopy is not indicated if no invasion of the airways is identified on EUS.
\end{abstract}

Presented at: NVGE/NVGIC (Dutch Society of Gastrointestinal Surgery), October 2007, Veldhoven the Netherlands (oral presentation); United European Gastroenterology Week, October 2007, Paris, France (poster presentation); European Society of Esophagology, September 2007, Dublin, Ireland (poster presentation).

Sources of financial support: JMT Omloo is supported by a grant from Zon Mw Health Care Efficiency Research (945-04-510).

J. M. T. Omloo $(\bowtie) \cdot$ M. van Heijl •

M. I. van Berge Henegouwen · J. J. B. van Lanschot

Department of Surgery, Academic Medical Center,

University of Amsterdam,

Meibergdreef 9,

1105 AZ Amsterdam, The Netherlands

e-mail: j.m.omloo@amc.uva.nl

\section{J. J. G. H. M. Bergman}

Department of Gastroenterology, Academic Medical Centre,

University of Amsterdam,

Amsterdam, The Netherlands
M. G. J. Koolen

Department of Pulmonary Diseases, Academic Medical Centre,

University of Amsterdam,

Amsterdam, The Netherlands

Present address:

J. J. B. van Lanschot

Department of Surgery, Erasmus Medical Centre,

Rotterdam, The Netherlands 
Keywords Esophageal cancer · Bronchoscopy Endoscopic ultrasonography $\cdot$ Staging

\section{Introduction}

Esophageal cancer is an aggressive disease with early lymphatic and hematogeneous dissemination, and a strong tendency to infiltrate into surrounding structures. Despite many improvements in diagnostic and therapeutic strategies, the prognosis is still unfavorable. ${ }^{1-4}$

The proximal part of the intrathoracic esophagus is located between the trachea and the vertebral column. Therefore, esophageal tumors at or above the carina tend to invade the tracheobronchial tree, precluding curative surgery. To assess local resectability endoscopic ultrasonography (EUS) is generally considered the most accurate modality as it is able to visualize distinct esophageal wall layers with an accuracy of more than $90 \% .^{5}$ Because of the great therapeutic consequences of tracheobronchial ingrowth, the preoperative workup of the patients with these proximal tumors frequently also includes bronchoscopy (with biopsy on indication) to exclude airway invasion. ${ }^{6,7}$ The usefulness of bronchoscopy in patients with proximal tumors has been investigated extensively, although not in relation to the accuracy of EUS to determine involvement of the airways. ${ }^{8,9}$

Therefore, the aim of the present study was to determine the additional value of preoperative bronchoscopy (with biopsy on indication) for detecting invasion of the tracheobronchial tree after having performed EUS in a specialized centre.

\section{Patients and Methods}

\section{Patients}

Patients visiting the outpatient clinic of our hospital for newly diagnosed esophageal cancer between January 1997 and December 2006 were included in this analysis. Eligible patients had histologically proven cancer of the upper and/ or middle thoracic esophagus. Patients were excluded if EUS or bronchoscopy was not performed and in case of subcarinal localization of the esophageal tumor.

\section{Endoscopic Ultrasonography}

A radial scanner (GF-UM130 or GF-UM160, 5-20 MHz, Olympus Medical Systems, Tokyo, Japan) was used for EUS. In case of a stenotic tumor that did not allow passage of the standard echo-endoscope, a small-caliber probe (Mh908, 7.5 MHz, Olympus Medical Systems, Tokyo, Japan) was used in an attempt to traverse the tumor. EUS was performed with the patient in a left decubitus position under conscious sedation using $2.5-10 \mathrm{mg}$ midazolam intravenously. All investigations were performed by or supervised by a gastroenterologist experienced in EUS.

A lesion was considered to invade the trachea on EUS if endosonographically the hyper-echoic interphase of the esophagus and trachea was interrupted. Close approximation of the tumor without such interruption was still considered compatible with T3 stage.

\section{Bronchoscopy}

Bronchoscopy was performed using a flexible videobronchoscope (BF-P160, Olympus Optical, Tokyo, Japan) via a transnasal approach after premedication with $2 \%$ lidocaine spray in nose and throat (up to $50 \mathrm{ml}$ ). During the examination $2 \%$ lidocaine spray was administered into the trachea and bronchi via the bronschoscope. No systemic medication was used. All investigations were performed by an experienced pulmonary physician. The complete tracheobronchial tree was inspected; laryngeal structures were included in the examination. All direct tumor signs (especially intraluminal growth and mucosal break) and indirect tumor signs (especially mobility of pars membranacea during coughing, bulging/ compression) were recorded. Mucosal brushing or biopsies were performed only if mucosal abnormalities were suspected.

No bronchoalveolar lavage was performed; however, rinsing fluid from brushing or biopsies was sent for cytologic examination.

\section{Other Investigations}

Computed tomography (CT) of the chest and abdomen and external ultrasonography of the neck were also performed in all patients to exclude distant metastases.

Endoscopic ultrasonography, CT, and external ultrasonography of the neck were performed in a random order; however, bronchoscopy was always performed after EUS. The bronschoscopist was aware of the other clinical data. If EUS showed a T3 tumor, while a T4 tumor was suspected on CT, EUS was used to determine the final $\mathrm{T}$ stage. All investigations were performed before any form of therapy was started.

\section{Neoadjuvant Therapy}

Due to the time span of this study, different neoadjuvant regimens were applied. In the first period, patients with squamous cell carcinoma received neoadjuvant chemotherapy consisting of two or four cycles of cisplatin and etoposide (depending on the tumor regression on $\mathrm{CT}$ after two cycles). In later years, patients received neoadjuvant chemoradiotherapy (five cycles of paclitaxel and carbopla- 
tin with concurrent radiation of 41.4 Gy) as part of a randomized clinical trial comparing surgery alone versus neoadjuvant chemoradiotherapy and surgery for squamous cell carcinoma and adenocarcinoma.

\section{Surgical Resection}

Because of the high localization of the tumor, in all patients who were considered eligible for potentially curative surgery, an esophagectomy was performed via the extended transthoracic approach with two field lymphadenectomy. A gastric tube was constructed and esophagogastrostomy was performed in the neck without cervical lymphadenectomy.

Tumor extent and airway invasion were assessed intraoperatively. If distant metastases and/or local irresectability due to invasion of vital structures was encountered, resection was abandoned.

\section{Statistical Analysis}

Tracheobronchial invasion on EUS, bronchoscopy (with positive biopsy results) and/or during operation was considered the gold standard for the presence of tracheobronchial invasion. No tracheobronchial invasion on EUS and/or bronchoscopy was considered as false negative if local irresectability was encountered during operation.

\section{Results}

\section{Clinicopathological Characteristics}

Between January 1997 and December 2006, a total of 106 patients presented at our outpatient clinic for analysis of a newly diagnosed histologically proven, esophageal malignancy at or above the carina. Two patients were excluded as EUS was not performed. The clinicopathological characteristics of all 104 patients are summarized in Table 1. The majority of patients were male (64 patients, $62 \%$ ) and the mean age was 61 years (range 32-85 years). Histology showed squamous cell carcinoma in 93 patients $(89 \%)$ and adenocarcinoma in 11 patients $(11 \%)$. Tumors were mainly localized in the middle thoracic part of the esophagus (75 patients, $72 \%$ ). A total of 98 patients (94\%) were referred from another hospital. In total, 24 patients $(23 \%)$ received neoadjuvant chemotherapy and six patients $(6 \%)$ received neoadjuvant chemoradiotherapy (of whom one patient was with adenocarcinoma). A complete surgical resection was performed in 49 patients $(47 \%)$, whereas in nine patients $(9 \%)$ resection was abandoned as local irresectability or distant metastases were encountered peroperatively. In 46 patients (44\%), the treatment was primarily palliative.
Table 1 Clinicopathological Characteristics of 104 Patients Visiting the Outpatient Clinic for Newly Diagnosed Histologically Proven Esophageal Cancer at or Above the Carina between January 1997 and December 2006

\begin{tabular}{lc}
\hline & Number of patients $(n=104)$ \\
\hline Gender & \\
M/F & $64 / 40(62 \% / 38 \%)$ \\
Age & $61(32-85)$ \\
Mean (range) [years] & \\
Histology & $93(89 \%)$ \\
Squamous cell carcinoma & $11(11 \%)$ \\
Adenocarcinoma & \\
Tumor localization & $7(7 \%)$ \\
Cervical esophagus & $22(21 \%)$ \\
Upper thoracic esophagus & $75(72 \%)$ \\
Middle thoracic esophagus & \\
Referral & $98(94 \%)$ \\
From other hospital & \\
Therapy & $24(23 \%)$ \\
Preoperative chemotherapy & \\
Preoperative chemoradiation & \\
Surgical resection & $6(6 \%)$ \\
Surgical exploration & $49(47 \%)$ \\
Primarily palliative treatment & $9(9 \%)$ \\
\hline
\end{tabular}

$M$ male, $F$ female

${ }^{\mathrm{a}}$ Two-four cycles of Cisplatin and Etoposide

${ }^{\mathrm{b}}$ Five cycles of paclitaxel and carboplatin with concurrent 41.4-Gy radiation

\section{Staging and Treatment}

Endoscopic ultrasonography described T-stages 1 to 3 in 78 patients (78/104, 75\%; Table 2, Fig. 1). EUS described local irresectability due to: invasion of the tracheobronchial tree in 12 patients $(12 / 104,12 \%)$, invasion of the aorta in six patients $(6 / 104,6 \%)$, invasion of the lung in 11 patients $(11 / 104,11 \%)$. In four patients invasion of multiple structures was seen: in two patients invasion of the aorta and the lung; in one patient invasion of the tracheobronchial tree and the lung; in one patient invasion of the tracheobronchial tree, the aorta, and the lung.

Bronchoscopy showed a fixed pars membranacea in five patients $(5 / 104,5 \%)$, bulging in 36 patients $(36 / 104,35 \%)$, and true invasion of the tracheobronchial mucosa (with positive biopsies) in only five of the patients $(5 / 104,5 \%)$. In five patients, more than one indirect and/or direct sign was seen; in three patients bulging and invasion, in one patient a fixed pars membranacea and bulging, and in one patient a fixed pars membranacea and bulging and invasion was seen.

Distant metastases were found in 12 patients $(12 / 104$, $12 \%)$. Eight patients were not suitable for surgery due to a poor general health condition $(8 / 104,8 \%)$. 
Table 2 Staging and Treatment of 104 Patients Visiting the Outpatient Clinic for Newly Diagnosed Histologically Proven Esophageal Cancer at or Above the Carina between January 1997 and December 2006

\begin{tabular}{lc}
\hline & Number of patients $(n=104)$ \\
\hline EUS & \\
T1-3 & $78(75 \%)$ \\
T4 & $26(25 \%)$ \\
Invasion tracheobronchial tree ${ }^{\mathrm{a}}$ & $12(12 \%)$ \\
Invasion aorta $^{\mathrm{a}}$ & $6(6 \%)$ \\
Invasion lung $^{\mathrm{a}}$ & $11(11 \%)$ \\
Other $^{\mathrm{b}}$ & $2(2 \%)$ \\
Bronchoscopy & \\
Fixed pars membranacea & $5(5 \%)$ \\
Bulging & $36(35 \%)$ \\
Invasion tracheobronchial tree & \\
Other contraindications for surgery & $5(5 \%)$ \\
Distant metastases & \\
Poor general health & $12(12 \%)$ \\
Treatment & $8(8 \%)$ \\
Potentially curative & \\
Primarily palliative & $58(56 \%)$ \\
Peroperative findings $(n=58)$ & $46(44 \%)$ \\
Surgical resection & \\
Surgical exploration/no resection & $49(84 \%)$ \\
Invasion tracheobronchial tree & $9(15 \%)$ \\
Invasion other vital structures & $5(8 \%)$ \\
Distant metastases & $2(3 \%)$ \\
\hline
\end{tabular}

EUS endoscopic ultrasonography

${ }^{a}$ In four patients invasion of multiple structures was seen; in two patients invasion of aorta and lung, in one patient invasion of tracheobronchial tree and lung and in one patient invasion of all three sites was seen

${ }^{\mathrm{b}}$ Other: invasion of right carotic artery in one patient, invasion of pericardium in one patient

${ }^{\mathrm{c}}$ In five patients more than one indirect and/or direct sign was seen; in three patients bulging and invasion, in one patient fixed pars membranacea and bulging, and in one patient all three signs were seen ${ }^{\mathrm{d}}$ Cyto- and/or histologically proven

After completion of the staging procedures, 58 patients were found suitable for potentially curative surgery $(56 \%)$. In nine patients $(9 / 58,15 \%)$ resection was abandoned peroperatively because of: invasion of the tracheobronchial tree (five patients, 5/58, 8\%), invasion of other vital structures (two patients, 2/58, 3\%) and distant metastases (two patients, 2/58, 3\%). Time between analysis and surgery did not differ between patients undergoing resection and in patients found to be irresectable peroperatively (median 2.9 months and 2.6 months, respectively).

\section{Additional Value of Bronchoscopy}

Indirect signs were seen on bronchoscopy in 40 patients (40/104, 38\%). Invasion of the tracheobronchial tree was diagnosed or encountered during operation in 17 patients
(17/104, 16\%) (Table 3). Indirect signs were seen during bronchoscopy in $29 \%$ of the patients (25/87) without invasion of the tracheobronchial tree (false positives), and no indirect signs were seen in $12 \%$ of the patients $(2 / 17)$ with invasion of the tracheobronchial tree (false negatives). This results in a sensitivity of $88 \%$ and a specificity of $71 \%$ of indirect signs seen on bronchoscopy reflecting invasion of the tracheobronchial tree.

\section{Discussion}

Preoperative staging in patients with esophageal cancer is an extensive diagnostic process. To detect incurable patients before surgery, various modalities are being used. Invasion of the airways is common in tumors located at or above the carina. Invasion of the tracheobronchial tree can be assessed by different diagnostic modalities, especially $\mathrm{CT}$, magnetic resonance imaging (MRI), EUS, and bronchoscopy. In the literature, CT and MRI both show low accuracy compared to EUS to determine T stage (45\% and $60 \%$, respectively). ${ }^{10,11}$ Therefore, it was investigated in the present study whether performing bronchoscopy after EUS has any additional value. In this study, bronchoscopy did not detect any patients with airway invasion that was not already detected by EUS. Remarkably, five patients $(5 / 58$, $8 \%$ ) were found to have airway invasion during surgery, which had been missed by both EUS and bronchoscopy.

Bronchoscopy is capable of directly detecting airway invasion if the tumor has breached the epithelium. If a wider definition of invasion would be used and indirect signs (esp. bulging and a fixed pars membranacea) would also be taken into account, the value of bronchoscopy might possibly rise. Baisi et al. evaluated invasion of the tracheobronchial tree in 91 patients with esophageal cancer by bronchoscopy. ${ }^{12}$ They concluded that compression of the tracheobronchial tree (bulging) does not necessarily indicate infiltration by the esophageal tumor; however, if also a fixed pars membranacea is seen, there is a frank infiltration, making radical resection highly unlikely. Riedel et al. have investigated 116 patients with bronchoscopy. ${ }^{13}$ Remarkably, they found microscopic proof of invasion in only $4 \%$ of patients showing indirect signs on bronchoscopy, whereas the remaining $96 \%$ of the patients underwent a radical resection. If in the present study, indirect signs were used as well, an unnecessary surgical exploration would have been prevented in two patients (12\%). However, 25 patients $(29 \%)$ would have been wrongly diagnosed with irresectable cancer.

Endoscopic ultrasonography via the esophagus examines the airways from an opposite angle, making it possible to detect airway invasion without a mucosal break, i.e. in an earlier stage. This fundamental difference could explain 
Figure 1 Flowchart of 104 patients visiting the outpatient clinic for newly diagnosed histologically proven esophageal cancer at or above the carina between January 1997 and December 2006. pts patients, $N O$ not potentially curable, YES potentially curable. Asterisk: In four patients invasion of multiple structures was seen; in two patients invasion of the aorta and the lung, in one patient invasion of the tracheobronchial tree and the lung, and in one patient invasion of the tracheobronchial tree, the aorta, and the lung was seen. Two asterisks, other: invasion of the right carotid artery in one patient, invasion of the pericardium and multiple metastatic lymph nodes in the neck, mediastinum, and abdomen in one patient.

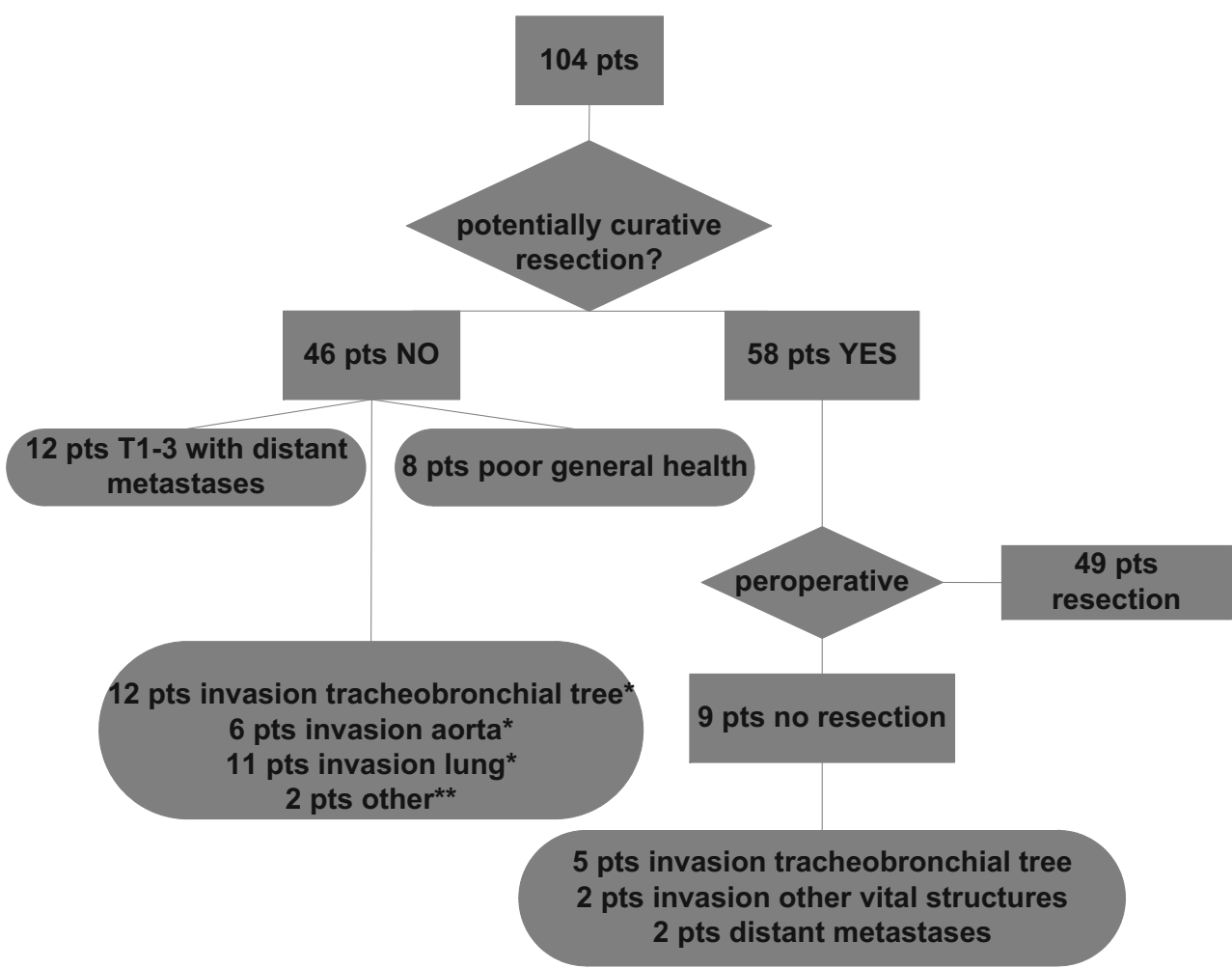

why EUS detects more patients with airway invasion compared with bronchoscopy. It should be realized, that EUS uses indirect signs as well in order to describe the T-stage and thus it is very much operator- and experiencedependent. Fockens et al. have described the prognosis of patients diagnosed with irresectable (T4) tumors on EUS, and found that these patients have a very poor prognosis, regardless of further therapy (including potentially curative surgery). ${ }^{14}$ In their analysis, 24 of 51 patients (47\%) underwent explorative surgery (despite EUS T-stage 4);

Table 3 Presence of Indirect Signs on Bronchoscopy of All 104 Patients

\begin{tabular}{lcc}
\hline & \multicolumn{2}{l}{ Number of patients $(\mathrm{n}=104)$} \\
\cline { 2 - 3 } & \multicolumn{2}{l}{ Invasion tracheobronchial tree } \\
\cline { 2 - 3 } & Present & Absent \\
\hline Indirect signs on bronchoscopy & 15 & 25 \\
Present & 2 & 62 \\
Absent & 17 & 87 \\
Total & & \\
\hline
\end{tabular}

"Indirect signs" implies a fixed pars membranacea, bulging and/or invasion of the tracheobronchial tree (cyto-/histologically proven). "Invasion tracheobronchial tree" implies tracheobronchial invasion on EUS, bronchoscopy (with positive biopsy results) and/or encountered during operation however, only 13 underwent a surgical resection, and in only three of these patients the resection was microscopically radical.

In the present study, bronchoscopic ultrasonography was not addressed. To our knowledge, only one study has compared esophageal ultrasonography, conventional bronchoscopy, and bronchoscopic ultrasonography for detecting airway invasion. ${ }^{15}$ Unfortunately, only $44 \%$ of the patients underwent esophageal ultrasonography due to stenosis. This study found accuracy rates for invasion of the airways of $91 \%$ with bronchoscopic ultrasonography, $78 \%$ with conventional bronchoscopy, and $85 \%$ with esophageal ultrasonography. The authors conclude that bronchoscopic ultrasonography is a safe and promising technique to determine local resectability. However, it should be taken into account that (bronchoscopic) ultrasonography is an invasive modality and the burden for the patient is relatively high. ${ }^{16-18}$

There are some limitations to the present study. The total number of patients is relatively small. Moreover, the percentage of patients in whom airway invasion was not detected until surgical exploration is relatively high. Time between analysis and surgery was not significantly longer compared with the patients who underwent resection.

In conclusion, bronchoscopy has no additional value in this study as a standard diagnostic modality for staging of patients with esophageal cancer at or above the carina after EUS in a specialized centre. Although based on small numbers, we 
conclude that bronchoscopy is not indicated if no invasion of the airways is identified on EUS. However, bronchoscopy (if possible in combination with bronchoscopic ultrasonography) should be performed if because of esophageal stenosis EUS is not feasible. If irresectability is identified during operation, bronchoscopy should rule out direct tumor invasion of the mucosa before radiotherapy is started, to prevent the development of a tracheo-esophageal fistula.

Open Access This article is distributed under the terms of the Creative Commons Attribution Noncommercial License which permits any noncommercial use, distribution, and reproduction in any medium, provided the original author(s) and source are credited.

\section{References}

1. Burmeister BH, Smithers BM, Gebski V et al. Surgery alone versus chemoradiotherapy followed by surgery for resectable cancer of the oesophagus: A randomised controlled phase III trial. Lancet Oncol 2005;6:659-668.

2. Hulscher JB, van Sandick JW, de Boer AG et al. Extended transthoracic resection compared with limited transhiatal resection for adenocarcinoma of the esophagus. $N$ Engl J Med 2002;347:1662-1669.

3. Lerut T, Coosemans W, Decker G et al. Cancer of the esophagus and gastro-esophageal junction: Potentially curative therapies. Surg Oncol 2001;10:113-122.

4. van Meerten E, Muller K, Tilanus HW et al. Neoadjuvant concurrent chemoradiation with weekly paclitaxel and carboplatin for patients with oesophageal cancer: A phase II study. Br J Cancer 2006;94:1389-1394.

5. Lightdale CJ, Kulkarni KG. Role of endoscopic ultrasonography in the staging and follow-up of esophageal cancer. J Clin Oncol 2005;23:4483-4489.
6. Allum WH, Griffin SM, Watson A, Colin-Jones D. Guidelines for the management of oesophageal and gastric cancer. Gut 2002;50 (Suppl 5):v1-v23.

7. Siersema PD, Rosenbrand CJ, Bergman JJ et al. Guideline 'Diagnosis and treatment of oesophageal carcinoma'. Ned Tijdschr Geneeskd 2006;150:1877-1882.

8. Baisi A, Bonavina L, Peracchia A. Bronchoscopic staging of squamous cell carcinoma of the upper thoracic esophagus. Arch Surg 1999;134:140-143.

9. Riedel M, Stein HJ, Mounyam L et al. Predictors of tracheobronchial invasion of suprabifurcal oesophageal cancer. Respiration 2000;67:630-637.

10. Marsman WA, Fockens P. State of the art lecture: EUS for esophageal tumors. Endoscopy 2006;38(Suppl 1):S17-S21.

11. Wu LF, Wang BZ, Feng JL et al. Preoperative TN staging of esophageal cancer: Comparison of miniprobe ultrasonography, spiral CT and MRI. World J Gastroenterol 2003;9:219-224.

12. Baisi A, Bonavina L, Peracchia A. Bronchoscopic staging of squamous cell carcinoma of the upper thoracic esophagus. Arch Surg 1999;134:140-143.

13. Riedel M, Hauck RW, Stein HJ et al. Preoperative bronchoscopic assessment of airway invasion by esophageal cancer: A prospective study. Chest 1998;113:687-695.

14. Fockens P, Kisman K, Merkus MP et al. The prognosis of esophageal carcinoma staged irresectable (T4) by endosonography. J Am Coll Surg 1998;186:17-23.

15. Nishimura Y, Osugi H, Inoue K et al. Bronchoscopic ultrasonography in the diagnosis of tracheobronchial invasion of esophageal cancer. J Ultrasound Med 2002;21:49-58.

16. Osugi $H$, Nishimura $Y$, Takemura $M$ et al. Bronchoscopic ultrasonography for staging supracarinal esophageal squamous cell carcinoma: Impact on outcome. World J Surg 2003;27:590-594.

17. Wakamatsu $T$, Tsushima $K$, Yasuo $M$ et al. Usefulness of preoperative endobronchial ultrasound for airway invasion around the trachea: Esophageal cancer and thyroid cancer. Respiration 2006;73:651-657.

18. Becker HD. EBUS: A new dimension in bronchoscopy. Of sounds and images - a paradigm of innovation. Respiration 2006;73:583586. 